Bundesgesundheitsbl 2013 · 56:1023-1027

DOI 10.1007/s00103-013-1782-6

๑) Springer-Verlag Berlin Heidelberg 2013
Richtlinien der Gendiagnostik-Kommission am Robert Koch-Institut

\section{Richtlinie der Gendiagnostik- Kommission (GEKO) für die Anforderungen an die Durchführung der vorgeburtlichen Risikoabklärung sowie an die insoweit erforderlichen Maßnahmen zur Qualitätssicherung gemäß § 23 Abs. 2 Nr. 5 GenDG}

\author{
in der Fassung vom 12.04.2013 \\ veröffentlicht und in Kraft getreten \\ am 22.04.2013
}

\section{Vorwort}

Am 01.02.2010 ist in Deutschland das Gesetz über genetische Untersuchungen bei Menschen (Gendiagnostikgesetz - GenDG) in Kraft getreten. Die Aufgabe, Richtlinien im gesetzlichen Rahmen ( $\$ 23 \mathrm{GenDG}$ ) für verschiedene Teilbereiche des GenDG zu erarbeiten, wurde der beim Robert Koch-Institut (RKI) eingerichteten Gendiagnostik-Kommission (GEKO) übertragen. Die GEKO ist aus 13 Sachverständigen aus den Fachrichtungen Medizin und Biologie, 2 Sachverständigen aus den Fachrichtungen Ethik und Recht sowie 3 Vertretern der für die Wahrnehmung der Interessen der Patientinnen und Patienten, der Verbraucherinnen und Verbraucher und der Selbsthilfe behinderter Menschen auf Bundesebene maßgeblichen Organisationen zusammengesetzt.

In $₫ 23$ Abs. 2 Nr. 5 GenDG wird festgelegt, dass die GEKO in Bezug auf den allgemein anerkannten Stand der Wissenschaft und Technik Richtlinien für die Anforderungen an die Durchführung der vorgeburtlichen Risikoabklärung sowie an die insoweit erforderlichen Maßnahmen zur Qualitätssicherung erstellt.

Die vorgeburtliche Risikoabklärung ist eine Untersuchung, mit der „die Wahrscheinlichkeit für das Vorliegen bestimmter genetischer Eigenschaften mit Bedeutung für eine Erkrankung oder gesundheitliche Störung des Embryos oder Fötus ermittelt werden soll" ( $\$ 3 \mathrm{Nr} .3$ GenDG). Eine solche Untersuchung erfolgt stets nach Aufklärung, entsprechender individueller Beratung und Einwilligung nach individueller Entscheidung der Schwangeren, die im Bewusstsein möglicher ethischer und psychosozialer Probleme gefällt wird und damit keinem Automatismus unterliegt.

\section{Anwendungsbereich der Richtlinie}

Vorgeburtliche genetische Untersuchungen sind entweder genetische Analysen zur Feststellung genetischer Eigenschaften ( $\$ 3$
Nr. 1a GenDG) oder vorgeburtliche Risikoabklärungen ( $\$ 3 \mathrm{Nr}$. 1b GenDG).

Bei der vorgeburtlichen Risikoabklärung wird mittels nichtinvasiver Methoden, insbesondere aus dem mütterlichen Alter sowie zusätzlichen sonographischen Parametern und laboratoriumsmedizinischen Messgrößen, ein schwangerschaftsspezifisches Risiko für das Vorliegen numerischer Chromosomenstörungen (Trisomie 21, 18 und 13, Monosomie X/Turner-Syndrom) des oder der Föten berechnet, für die ein entsprechender Algorithmus vorliegt. Das Ergebnis der Risikoberechnung wird in Form einer Rate angegeben, d. h. im Mittel ist 1 von $\mathrm{x}$ Schwangerschaften mit dem gleichen Testergebnis betroffen.

Im Gegensatz dazu erfolgt durch vorgeburtliche genetische Analysen z. B. mittels Karyotypisierung oder nichtinvasiver Pränataldiagnostik an fetaler DNA aus mütterlichem Blut (NIPD) der Ausschluss bzw. die Feststellung einer numerischen oder strukturellen Chromosomenstörung. Eine solche genetische 
Analyse zur Feststellung genetischer Eigenschaften, die nicht Gegenstand dieser Richtlinie ist, kann sich der vorgeburtlichen Risikoabklärung anschließen, ihr vorausgehen oder könnte sie ersetzen.

Die Vorsorgeuntersuchung von gesunden Schwangeren ohne erkennbare Risiken unter Einsatz der Ultraschalldiagnostik ist in den Mutterschafts-Richtlinien [1] verankert und sieht sonographische Untersuchungen um 10, 20 und 30 Schwangerschaftswochen vor. Diese Vorsorgeuntersuchungen werden vom GenDG nicht erfasst und intendieren daher keine Form der vorgeburtlichen Risikoabklärung im Sinne dieser Richtlinie, auch wenn hierbei zufällig Hinweiszeichen für eine genetische Erkrankung gefunden werden können.

\section{Ziel der vorgeburtlichen Risikoabklärung}

Ziel der vorgeburtlichen Risikoabklärung ist es, "lediglich die Wahrscheinlichkeit für das Vorliegen bestimmter genetischer Eigenschaften mit Bedeutung für eine Erkrankung oder gesundheitliche Störung beim ungeborenen Kind, z. B. Chromosomenstörungen , ${ }^{1}$ abzuklären. Die Schwangere soll damit in die Lage versetzt werden, eine individuelle Entscheidung für oder gegen eine invasive vorgeburtliche genetische Untersuchung treffen zu können. Im Vergleich zur früheren Ermittlung des Risikos allein aus dem mütterlichen Alter wird durch die individuelle vorgeburtliche Risikoabklärung die Zahl invasiver Untersuchungen ohne Verringerung der Entdeckungsrate reduziert [2].

Voraussetzung für die vorgeburtliche Risikoabklärung sind eine kompetente Aufklärung und genetische Beratung, insbesondere die auf den Einzelfall abgestimmte, klare und Missverständnisse vermeidende Risikokommunikation. Die Anforderungen an die genetische Beratung sind in einer gesonderten Richtlinie der GEKO geregelt [3].

1 BT-Drucks. 16/10532, S. 21.

\section{Vorbemerkung zur Methode}

Die vorgeburtliche Risikoabklärung muss dem allgemein anerkannten Stand der Wissenschaft und Technik entsprechen. Sie erfolgt derzeit als Ersttrimesteruntersuchung - in der Regel zwischen 11 und 14 abgeschlossenen Schwangerschaftswochen (SSW) p.m. (post menstruationem) [4, 5, 6, 7]. Zur Anwendung kommen derzeit: die Anamnese mütterlicher Risikofaktoren, die Ultraschalluntersuchung mit Messung der Scheitel-Steiß-Länge und der fetalen Nackentransparenz sowie optional zusätzliche sonographisch festgestellte morphologische oder hämodynamische Parameter oder strukturelle Besonderheiten und die Bestimmung serologischer laboratoriumsmedizinischer Messgrößen, wie der freien $\beta$-Kette des humanen Choriongonadotropins (freies $\beta$-hCG) und das schwangerschaftsassoziierte Plasmaprotein A (PAPP-A) aus mütterlichem Blut. Aus den Ergebnissen der anamnestischen, sonographischen oder laboratoriumsmedizinischen Untersuchungen werden mithilfe von Algorithmen individuelle Wahrscheinlichkeiten berechnet $[8,9]$.

\section{Anforderungen}

\section{V.1 Voraussetzungen für die vorgeburtliche Risikoabklärung}

Die verantwortliche ärztliche Person klärt unter Berücksichtigung der Anamnese entsprechend $\$ 9$ GenDG und der „Richtlinie der Gendiagnostik-Kommission für die Anforderungen an die Inhalte der Aufklärung bei genetischen Untersuchungen zu medizinischen Zwecken gemäß $\$ 23$ Abs. 2 Nr. 3 GenDG“ [10] die Schwangere insbesondere über den Unterschied zwischen einer vorgeburtlichen Risikoabklärung und einer genetischen Analyse auf. Der Schwangeren muss vermittelt werden, dass die meisten auffälligen (d. h. positiven) Testergebnisse falsch positiv im Hinblick auf das tatsächliche Vorliegen einer numerischen Chromosomenstörung sind. Denn ein auffälliges (Test-positives) Untersuchungsergebnis im Rahmen der vorgeburtlichen Risikoabklärung ist dadurch definiert, dass z. B. ein Risiko von mehr als 1:250 für ein Kind mit Trisomie 21 besteht.

Über Wesen, Bedeutung und Tragweite der vorgeburtlichen Risikoabklärung einschließlich möglicher auftretender ethischer Dilemmata und psychosozialer Probleme sowohl bei Vorliegen falsch positiver als auch richtig positiver Befunde ist aufzuklären.

Es bedarf der schriftlichen Einwilligung der Schwangeren nach $₫ 8$ Abs. 1 GenDG.

Eine genetische Beratung muss vor und nach der vorgeburtlichen Risikoabklärung entsprechend $\$ 10$ Abs. 2 und 3 GenDG und nach der „Richtlinie der Gendiagnostik-Kommission über die Anforderungen an die Qualifikation zur und Inhalte der genetischen Beratung gemäß $\$ 23$ Abs. 2 Nr. 2a und $\$ 23$ Abs. 2 Nr. 3 GenDG“ [3] erfolgen und einen Hinweis auf den Beratungsanspruch nach $₫ 2$ Schwangerschaftkonfliktgesetz [11] enthalten. Der Inhalt der Beratung ist nach $\$ 15$ Abs. 3 GenDG zu dokumentieren.

\section{V.2 Durchführung der vorgeburtlichen Risikoabklärung}

Vorgeburtliche Risikoabklärungen müssen in der diagnostischen Kette eine hohe, dem allgemein anerkannten Stand der Wissenschaft und Technik entsprechende Qualität aufweisen. Diese wird maßgeblich bestimmt durch umfassende Kenntnisse und Fertigkeiten der ärztlichen Person in der Durchführung der Diagnostik, die Validität und Güte des angewandten Algorithmus und die kompetente Interpretation der Ergebnisse sowie die Güte der genetischen Beratung.

Die verantwortliche ärztliche Person stellt sicher, dass die sonographischen, laboratoriumsmedizinischen und ggf. weiteren Einzelergebnisse zusammengeführt, interpretiert und mitgeteilt werden.

Als technische Voraussetzungen sind insbesondere Ultraschallgeräte und die eingesetzte Software qualitätsrelevant. Die Qualität aller diagnostischen Schritte ist regelmäßig zu sichern (s. Abschnitt V.5 dieser Richtlinie). Maßnahmen der externen Qualitätssicherung müssen 
den Vorgaben des GenDG und dieser Richtlinie entsprechen. Die Anbieter der Dienstleistungen, z. B. von Leistungspaketen, die zur Berechnung des Risikos dienen, müssen die Richtlinienkonformität ihrer Leistungen nachweisen.

\section{V.3 Geräte, laboratoriums- medizinische Untersuchungen und Algorithmus}

\section{V.3.1 Geräte}

Die Geräte müssen geeignet sein, die in dieser Richtlinie festgelegten Qualitätsanforderungen an die Untersuchungen zu erfüllen. Bezogen auf die Messung der Nackentransparenz ist eine Auflösung des Ultraschallgerätes erforderlich, die eine Unterscheidung um $0,1 \mathrm{~mm}$ ermöglicht. ${ }^{2}$

\section{V.3.2 Laboratoriumsmedizinische Untersuchungen}

Fachlich verweist die GEKO hinsichtlich der Qualitätssicherung der laboratoriumsmedizinischen Untersuchungen $z$. B. von $\beta$-hCG und PAPP-A auf die Regelungen des Medizinprodukterechts einschließlich der entsprechenden Richtlinie der Bundesärztekammer [12]. Die Entnahme des Untersuchungsmaterials für die laboratoriumsmedizinischen Untersuchungen kann nach aktuell anerkanntem Stand der Wissenschaft und Technik ab 9 SSW p.m. erfolgen. Die Anforderungen an die Qualitätssicherung genetischer Analysen zu medizinischen Zwecken gemäß $\$ 23$ Abs. 2 Nr. 4 GenDG sind in einer gesonderten Richtlinie der GEKO geregelt [13].

\section{V.3.3 Algorithmus}

Der Algorithmus und die Software, die für die Risikoberechnung verwendet werden, müssen diesen Zweck nachgewiesen erfüllen, d. h., sie müssen nach-

\footnotetext{
2 Die axiale Auflösung von bildgebenden Ultraschallgeräten hängt von der verwendeten Wellenlänge ab. Sie lässt aus physikalischen Gründen bei den verwendeten Frequenzen und entsprechenden Wellenlängen eine Differenzierung von maximal $0,1 \mathrm{~mm}$ zu.
}

vollziehbar, wissenschaftlich begründet ${ }^{3}$ und im Peer-Review-Verfahren ${ }^{4}$ publiziert sein. Die verwendete Software muss validiert sein. $^{5}$

Die Berechnung des Risikos bei Ersttrimesteruntersuchungen beruht aktuell auf Scheitel-Steiß-Länge, mütterlichem Alter, fetaler Nackentransparenz und maternalem freiem $\beta$-hCG sowie PAPP-A. In den Algorithmus können bei hinreichender Evidenzbasis zusätzliche Einflussgrößen wie z. B. Gestationsalter, maternales Gewicht, Ethnizität, Zigarettenrauchen, Parität und Konzeptionsmodus sowie Chorionizität bei Zwillingen einbezogen werden. Zur Anwendung sollten nur Parameter und Algorithmen kommen, die zusammen eine Entdeckungsrate von derzeit mindestens 85\% und eine Falsch-positiv-Rate von derzeit maximal 5\% erreichen.

Mit der verwendeten Software müssen alle für die individuelle Risikoberechnung relevanten Daten gespeichert werden können, damit eine unabhängige Überprüfung des Ergebnisses möglich ist.

\footnotetext{
3 Gefordert wird die Veröffentlichung des gesamten Algorithmus mit detaillierter Soft30) richtig positiv wären (s. auch Appendix, - Tab. 1, A Abb. 1).
} warespezifikation, die präzise und nachvollziehbar beschreibt, wie die Risiken berechnet werden.

${ }^{4}$ Unabhängig vom Peer-Review muss die Studie eine Reihe von Qualitätskriterien erfüllen, u. a. eine hohe Fallzahl, eine hohe Rücklaufquote, die detaillierte Beschreibung der Population und die entsprechende Qualifikation der daran beteiligten Ultraschalldiagnostiker.

${ }^{5}$ Da es sich um eine Software für diagnostische Zwecke und somit um ein Medizinprodukt handelt, ist das MPG zu berücksichtigen. Die verwendete Software muss entsprechend validiert sein. Bei der Eigenherstellung, das gilt auch für Software zu diagnostischen und therapeutischen Zwecken, wird der Betreiber zum Hersteller. Die Validierung von Medizinprodukten aus Eigenherstellung ( $\$ 3 \mathrm{Nr} .21 \mathrm{MPG}$ ) ist in den $\S \S 7,12$ und 19 des Gesetzes über Medizinprodukte (MPG) [14] geregelt. Die Beschreibung von Inhalt und Umfang der Validierung findet sich in Anhang I "Grundlegende Anforderungen" Richtlinie 98/79/EG des Europäischen Parlaments und des Rates über in-vitroDiagnostika (IVDD) [15].

\section{Beispielrechnung für die vor- geburtliche Risikoabklärung der Trisomie 21 (85\% Entdeckungs- rate und $5 \%$ Falsch-positiv-Rate bei einem Schwellenwert von $1: 250$ )}

Für die Interpretation des Ergebnisses einer vorgeburtlichen Risikoberechnung ist der gesetzte Schwellenwert für das Risiko von Bedeutung, da dieser die Sensitivität und Spezifität des Tests festlegt. Bei Einführung der vorgeburtlichen Risikoabklärung in den frühen 70er-Jahren wurde das Altersrisiko einer 35-Jährigen (1:250) als Schwellenwert festgelegt [16], d. h., ein Risiko von 1:250 oder größer ist gleichbedeutend mit einem auffälligen Testergebnis. In dem folgenden Beispiel ist der Algorithmus so ausgelegt, dass bei Untersuchung aller Schwangeren 85\% der Trisomie-21-Fälle ein auffälliges Testergebnis liefern würden (Sensitivität bzw. „Entdeckungsrate" der vorgeburtlichen Risikoabklärung). Bei einer Prävalenz der Trisomie 21 von 1:500 unter allen Schwangeren in Deutschland lägen bei 100.000 getesteten Schwangerschaften im Mittel 200 mit einer Trisomie 21 vor. Von diesen würden 170 (85\%) entdeckt, und 30 Fälle blieben unentdeckt.

Nimmt man an, der gewählte Schwellenwert (1:250) führt zu einer Rate von $5 \%$ falsch positiven Testresultaten, so wäre unter den 100.000 getesteten mit $4990(0,05 \times 99.800)$ auffälligen Schwangerschaften ohne Trisomie 21 zu rechnen. Insgesamt sind somit im Mittel 5160 auffällige Testergebnisse zu erwarten, von denen 3,3\% (etwa 1 von

\section{V.4 Berichtsqualität}

Im schriftlichen Befund müssen die $\mathrm{Pa}$ tientendaten, anamnestischen Daten und die patientenbezogenen Messergebnisse, Angaben zum verwendeten Algorithmus und zur Software sowie die berechneten Ergebnisse der vorgeburtlichen Risikoabklärung enthalten sein. 


\section{V.5 Maßnahmen der Qualitätssicherung der vorgeburtlichen Risikoabklärung}

Die Durchführung qualitätssichernder Maßnahmen setzt als Kenntnisinhalte und Fertigkeiten voraus:

- fetale Chromosomenstörungen (Trisomien 21, 18, 13, Monosomie X/ Turner-Syndrom, Triploidie, genetische Syndrome),

- Technik der Nackentransparenzmessung,

- sonographische, morphologische und hämodynamische ErsttrimesterMarker,

- laboratoriumsmedizinische Indikatoren, Einfluss- und Korrekturfaktoren für Ersttrimester-Untersuchungen.

\section{V.5.1 Kontinuierliche Qualitätssicherung}

Die ärztliche Person muss ihre Resultate einer externen, regelmäßigen Qualitätssicherung aller diagnostischen Schritte (Ultraschall, laboratoriumsmedizinische Untersuchungen, Algorithmus sowie Gesamtperformance) unterziehen. Im Falle der Ultraschallmessung ist die Messwerteverteilung für die jeweiligen ärztlichen Personen zu dokumentieren und jährlich mit Referenzwerten abzugleichen.

\section{Begründung}

Die hier aufgeführten Anforderungen an die Durchführung der vorgeburtlichen Risikoabklärung sowie an die insoweit erforderlichen Maßnahmen zur Qualitätssicherung sind international anerkannt. Die vorgeburtliche Risikoabklärung insgesamt sowie jede einzelne ihrer Komponenten (Ultraschall, laboratoriumsmedizinische Untersuchungen, Algorithmus) müssen einer kontinuierlichen Qualitätssicherung unterzogen werden. Durch eine kontinuierliche Qualitätssicherung kann das Problem der Unterschätzung der Nackentransparenz deutlich verringert und so die Entdeckungsrate erhöht werden [17, 18, 19]. Eine externe Qualitätssicherung ist insbesondere für den Bereich Ultraschall wesentlich, weil es sich bei der fetalen Nackentransparenz um eine Mes- sung im Bereich um 0,1 $\mathrm{mm}$ handelt und deren Genauigkeit direkt von der Qualifikation des Anwenders abhängt [17]. Eine regelmäßige jährliche Überprüfung der Messergebnisse und der Ultraschallbilder dient dem Absichern präziser Ergebnisse.

Eine kompetente Risikokommunikation, im Rahmen derer zum einen die Entdeckungsrate der Methode sowie die Falsch-positiv- und Falsch-negativ-Raten und der positiv prädiktive Wert als auch resultierende ethische Probleme vermittelt werden, soll der vorgeburtlichen Risikoabklärung vorausgehen, damit deren Ergebnisse von der Schwangeren verstanden werden.

\section{Inkrafttreten}

Diese Richtlinie wird nach Verabschiedung ihrer endgültigen Form durch die GEKO mit der Veröffentlichung auf der Homepage des RKI wirksam.

\section{Literatur}

1. Gemeinsamer Bundesausschuss (2013) Richtlinien des Bundesauschusses der Ärzte und Krankenkassen über die ärztliche Betreuung während der Schwangerschaft und nach der Entbindung („Mutterschafts-Richtlinien“). - zuletzt geändert am 20. Dezember 2012. http://www.g-ba.de/ downloads/62-492-678/Mu-RL_2012-12-20.pdf (Zugegriffen: 05.04.2013)

2. Hagen A, Entezami M, Gasiorek-Wiens A et al (2011) The impact of first trimester screening and early fetal anomaly scan on invasive testing rates in women with advanced maternal age. UItraschall Med 32:302-306

3. Richtlinie der Gendiagnostik-Kommission (GEKO) über die Anforderungen an die Qualifikation zur und Inhalte der genetischen Beratung gemäß 23 Abs. 2 Nr. $2 a$ und 23 Abs. 2 Nr. 3 GenDG. http:// www.rki.de/DE/Content/Kommissionen/GendiagnostikKommission/Richtlinien/Richtlinien_node.html (Zugegriffen: 05.04.2013)

4. Snijders RJ, Noble P, Sebire N et al (1998) UK multicentre project on assessment of risk of trisomy 21 by maternal age and fetal nuchal-translucency thickness at 10-14 weeks of gestation. Lancet 352:343-346

5. Gasiorek-Wiens A, Tercanli S, Kozlowski P et al (2001) Screening for trisomy 21 by fetal nuchal translucency and maternal age: a multicenter project in Germany, Austria and Switzerland. UItrasound Obstet Gynecol 18:645-648

6. Wright D, Kagan KO, Molina FS et al (2008) A mixture model of nuchal translucency thickness in screening for chromosomal defects. Ultrasound Obstet Gynecol 31:376-383

7. Kagan KO, Etchegaray A, Zhou Y et al (2009) Prospective validation of first-trimester combined screening for trisomy 21. Ultrasound Obstet Gynecol 34:14-18
8. Merz E, Thode C, Alkier A et al (2008) A new approach to calculating the risk of chromosomal abnormalities with first-trimester screening data. Ultraschall Med 29:639-645

9. Merz E, Thode C, Eiben B et al (2011) Individualized correction for maternal weight in calculating the risk of chromosomal abnormalities with first-trimester screening data. Ultraschall Med 32:33-39

10. Richtlinie der Gendiagnostik-Kommission (GEKO) für die Anforderungen an die Inhalte der Aufklärung bei genetischen Untersuchungen zu medizinischen Zwecken gemäß § 23 Abs. 2 Nr. 3 GenDG. - zuletzt geändert am 16.11.2012. http://www.rki. de/DE/Content/Kommissionen/GendiagnostikKommission/Richtlinien/Richtlinien_node.html (Zugegriffen: 05.04.2013)

11. Gesetz zur Vermeidung und Bewältigung von Schwangerschaftskonflikten (Schwangerschaftskonfliktgesetz - SchKG). http://www.gesetzeim-internet.de/beratungsg/ (Zugegriffen: 05.04.2013)

12. Bundesärztekammer (2011) Richtlinie der Bundesärztekammer zur Qualitätssicherung laboratoriumsmedizinischer Untersuchungen. Dtsch Arztebl 108(43):A-2298/B-1938/C-1918. http:// www.aerzteblatt.de/pdf.asp?id=111112 (Zugegriffen: 05.04.2013)

13. Richtlinie der Gendiagnostik-Kommission (GEKO) für die Anforderungen an die Qualitätssicherung genetischer Analysen zu medizinischen Zwecken gemäß § 23 Abs. 2 Nr. 4 GenDG. http://www.rki. de/DE/Content/Kommissionen/GendiagnostikKommission/Richtlinien/Richtlinien_node.html (Zugegriffen: 05.04.2013)

14. Gesetz über Medizinprodukte (Medizinproduktegesetz - MPG). http://www.gesetze-im-internet. de/bundesrecht/mpg/gesamt.pdf (Zugegriffen: 05.04.2013)

15. Europäisches Parlament, Rat der Europäischen Union (1998) Richtlinie 98/79/EG des Europäischen Parlaments und des Rates über In-vitroDiagnostika. ABI L 41(331):1-37. http://eurlex.europa.eu/LexUriServ/LexUriServ.do?uri= OJ:L:1998:331:0001:0037:DE:PDF (Zugegriffen: 05.04.2013)

16. Nicolaides KH (2011) Screening for fetal aneuploidies at 11 to 13 weeks. Prenat Diagn 31:7-15

17. Snijders RJ, Thom EA, Zachary JM et al (2002) First-trimester trisomy screening: nuchal translucency measurement training and quality assurance to correct and unify technique. Ultrasound Obstet Gynecol 19:353-359

18. Palomaki GE, Neveux LM, Donnenfeld A et al (2008) Quality assessment of routine nuchal translucency measurements: a North American laboratory perspective. Genet Med 10:131-138

19. Koster MP, Wortelboer EJ, Engels MA et al (2009) Quality of nuchal translucency measurements in The Netherlands: a quantitative analysis. Ultrasound Obstet Gynecol 34:136-141 


\section{APPENDIX}

Tab. 1 Erwartete Ergebnisse der Risikoberechnung für Trisomie 21 mittels Ersttrimesteruntersuchung (100.000 Schwangerschaften)

\begin{tabular}{llll} 
Testergebnis & \multicolumn{2}{l}{ Status der Schwangerschaft } & \multirow{2}{*}{ Alle } \\
\cline { 2 - 3 } & Trisomie 21 & Keine Trisomie 21 & \\
\hline Auffällig $(\geq 1 / 250)$ & $170(85,00 \%)^{\mathrm{a}}$ & $4990(5,00 \%)^{\mathrm{b}}$ & 5160 \\
& $3,29 \%^{\mathrm{c}}$ & & \\
\hline Unauffällig $(<1 / 250)$ & $30(15,00 \%)^{\mathrm{d}}$ & $94.810(95,00 \%)^{\mathrm{e}}$ & 94.840 \\
& & $99,97 \%^{\mathrm{f}}$ & \\
\hline Alle & $200^{\mathrm{g}}$ & 99.800 & 100.000 \\
\hline
\end{tabular}

aSensitivität („Entdeckungsrate") des Tests. 'Falsch-positiv-Rate des Tests. 'Anteil unter allen 5160 auffälligen

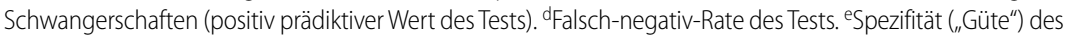
Tests. ${ }^{\mathrm{f}}$ Anteil unter allen 94.840 unauffälligen Schwangerschaften (negativ prädiktiver Wert des Tests). ${ }^{9}$ Entsprechend 1:500, d. h. der Prävalenz der Trisomie 21 bei allen Schwangeren in Deutschland.

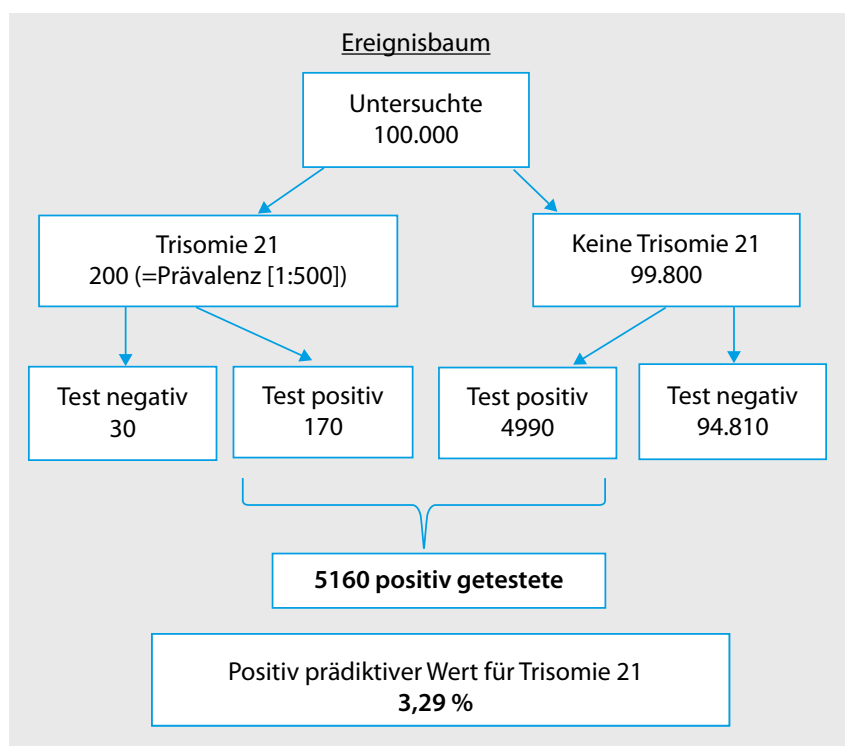

Abb. $1<$ Positiv prädiktiver Wert für Trisomie 21 